\title{
Online Social and Financial Management Medium for Non-business Enterprises
}

\author{
Alexandre Cunha ${ }^{1}$, Fernando Almeida ${ }^{2}$ \\ ${ }^{1}$ Higher Polytechnic Institute of Gaya, ISPGaya \\ V. N. Gaia, Portugal \\ ${ }^{2}$ University of Porto, INESC TEC \& ISPGaya \\ Porto, Portugal
}

\begin{abstract}
Online medium has greater influence in the last few decades as the people associated with the system use it for transactions including financial ones. In the online medium the participants use a variety of tools and resources to communicate and network. Such online medium is used for many activities out of which the financial contribution to the enterprises is one where the online tool is much used. To solve and propose a workable medium we have developed a system which can transact financial resources and goods. The proposed medium will allow the transfer of money and goods using the tools and enable to disable the publishing of data about it and observe the transactions. This design is based on a well-developed architecture and promote the use.
\end{abstract}

Keywords: Online Interfaces, Web Medium, Financial Network, Non-business Enterprise

Received: 13 May 2019, Revised 28 June 2019, Accepted 3 July 2019

DOI: $10.6025 / \mathrm{ijwa} / 2019 / 11 / 3 / 97-109$

(C) 2019 DLINE. All Rights Reserved

\section{Introduction}

The social economy is an economic and social force that opens the doors to another way of producing and consuming, respectful of people and the environment. It constitutes a heterogeneous socioeconomic space, formed by a set of socioeconomic activities, freely carried out by several entities with different organic realities, in their form, size and logic of operation.

In this institutional domain, there are cooperatives, mutual societies, mercies, associations, foundations and community entities, which have as common denominator organizations of persons that pursue the general interest or socially relevant interests of their members or beneficiaries in a sustainable way, and without the purpose of remunerating capitalist investors. Its specificity lies not in the object of the activity, because the social economy is present in almost all economic activities, from agriculture to services, but in ends, the processes, mode of organization, government and functioning, values and principles which guide their activities are common among them.

The social economy proves it is conceivable to combine profitability with solidarity, to create stable and dignified jobs, to

International Journal of Web Applications Volume 11 Number 3 September 2019 
strengthen social, economic and regional cohesion, to generate human and social capital, to promote active citizenship, to promote social, environmental and technological sustainability and innovation. The social economy is important in itself, but also because of the beneficial effects it generates. It is relevant to emphasize that the place of the social economy depends on its own actors, but also, to a large extent, on the vision and attitude of the political power and, consequently, on the public policies that are directed to it.

The weight of the social economy is growing throughout the European Union (EU). According to European Commission (2017), there are 2 million of social economy enterprises in Europe, representing $10 \%$ of all businesses in the EU. In Portugal, the social economy is also of significant importance, contributing approximately $4 \%$ to the national GDP. More than 260 thousand workers are dedicated to activities related to the social economy with more than 55 thousand entities involved (Ramos, 2016; Tiago, 2017).

This study is motivated by the dramatic events that occurred in the summer of 2017 in Portugal, in which fires caused more than 100 deaths (ABC, 2017). In accordance to this emergence scenario, a set of initiatives of solidarity at national and international level appeared, but they showed several difficulties in the coordination of the goods needed by the populations. In this sense, we propose the development of a digital donation platform that can help nonprofit and charity organizations in the process of identifying and collecting the necessary goods needed by populations. The digital application allows solidarity institutions to register and show their humanitarian and social causes. Users will be able to support these causes with the necessary assets identified by those organizations, according to the needs of each of these causes. The paper is organized as follows: we initially perform a brief contextualization of the project framework describing the growing importance that the Internet has had in the process of incentive and management of the donations. Next, we present the methodology of work, in which we present the requirements and architecture of the system and also the research questions. After that, we discuss the main results of the project considering each of the research questions identified in the previous point. Finally, we present the conclusions of our work.

\section{Background}

Technology can play a relevant role in the context of the nonprofit sector. According to Boles (2013), technological innovations can contribute to the increase of efficiency and effectiveness of these organizations, emphasizing the role of cloud computing systems, social media, and mobile technologies. Hackler \& Saxton (2007) consider that IT can contribute in the areas of financial sustainability, strategic communications, relationship building and partnerships. Mirza (2010) presents a study that intends to understand the critical success factors in the development of an information system for a charitable organization. This study emphasizes that the process of requirements analysis is fundamental since the budget of these institutions is typically very small and the proactive role that must be assumed by the promoters of these institutions in supporting the project.

Donations can take several types. Leskovec (2010) presents three typologies: (i) voluntary payments; (ii) monetary donations; and (iii) donation of goods. In the case of voluntary payments, the initiative of this action is carried out by the donor, while in monetary or property donations there is a contextualization of the cause to which the donation is destined. In any of these situations, Leskovec (2010) emphasizes the role that online environments can play in this process, namely in strengthening the relationship between charitable institutions and donors.

The role of the Internet for the charitable fundraising is addressed by several authors. Days (2009) and Hagenbuch (2007) discuss how the Internet has transformed the way that nonprofit organizations raising funds. Treiblmaier \& Pollach (2008) characterize the driver and inhibitors of online donations reaching the conclusion that fourth characteristic influences general attitude towards online donations, respectively: (i) pertinence and urgency of the cause; (ii) credibility of the organization; (iii) trust in the Internet; and (iv) perception about privacy. In this sense, studies have emerged that address the performance of online fundraising and the role that marketing plays in this process (Sargeant et al., 2007; Bennett, 2009; Cacija, 2016). Another factor, highlighted by Tatham \& Christopher (2014), is the role that logistics play in the Internet donation process, particularly when it comes to donating goods.

The growth of the strategic importance of social media and the increasing number of its users has also raised the motivation to observe this phenomenon in the third sector. Nah \& Saxton (2013) seek to understand the process of adoption and use of social media by nonprofit organizations. The perspective of analyzing the use of the main social networks, as Twitter and Facebook took into account three dimensions: (i) adoption; (ii) frequency of use; (iii) and dialogue. Some organizations in the third sector

\begin{tabular}{lllllll}
\hline 98 & International Journal of Web Applications & Volume & 11 & Number 3 & September 2019 \\
\hline
\end{tabular}


have also sought to gather a set of indications and suggestions for the use of social networks. At this level, a proposal for the collection of good practices for nonprofit organizations carried out by the Canadian Coalition for Global Health Research (CCGHR) should be enumerated. This study highlights the main benefits brought by the use of social networks and establishes a set of ethical guidelines for its use. In terms of benefits, the following stand out (Cole, 2014): (i) better interaction with supporters; (ii) drive traffic, which increases visibility; (iii) improve results; (iv) capture interest of the visitors; (v) increase message spread; (vi) influence supporters; (vii) enable grassroots movements; (viii) humanize the organization; (ix) acquire new contacts; and (x) increase trust and loyalty. The suggested guidelines are (Cole, 2014): (i) be fair and prudent; (ii) avoid deception; (iii) maintain dignity and respect; (iv) eschew secrecy; (v) be reversible; (vi) be transparent; (vii) clearly identify; (viii) rational analysis; (ix) emphasize clarity; (x) disclose; (xi) verify sources and data; (xii) establish responsibility; (xiii) examine intention; (xiv) encourage the good; and (xv) consistency builds trust. Finally, it is important to mention the study developed by Warner et al. (2014) in which they emphasize five social media best practices for nonprofit organizations: (i) listening to the audience; (ii) engaging instead of fundraising; (iii) sharing relevant and actionable content; (iv) consider the use of social media as part of a multi-channel strategy; and (v) measure the process.

The role of social networks in the communication process is also addressed considering the technological potential of each social network. Tugrul \& Lee (2018) mention that social networks allow people to be more connected with social causes, and one of the preponderant elements in people's participation is their physical and ideological proximity. Microblogging is referred by Lovejoy \& Saxton (2012) as one of the elements that enhance the exchange of information among several stakeholders of a nonprofit organization. The study by Lovejoy \& Saxton (2012) examined the practices of 100 largest nonprofit organizations in the United States to conclude that Twitter is strategically adopted by these organizations to promote greater dialogue and foster community-building practices. On the other hand, Lucas (2017) emphasizes the role that Facebook can play in strengthening relationships with supports, namely by encouraging social interaction and humanizing the brand.

Mobile technologies have been another element that has originated behavioral changes in society. Schwedel (2013) points out that mobile technology has led donors to prefer instant communication models and desire to get immediate answers to their requests. Moraru (2014) states that nonprofit organizations are adopting mobile technologies in different activities, such as administrative efficiency, SMS campaigns and innovation in fundraising activities. Choi \& Kim (2016) advocate that smartphones transformed the donation activity, making them more convenient without interfering in users' daily schedules. This study found that four attributes have significant positive impacts on users' willingness to use mobile donation applications, namely; (i) typecause fit, (ii) name-cause fit, (iii) cause involvement, and (iv) perceived effectiveness.

Despite their unequivocal importance in the context of the social development of the society, the proposals for creating webbased information system to support the donation activities of nonprofit organizations have been a very little explored area. This situation is confirmed in the study developed by Umapathy \& Huang (2015), which refer that most nonprofit organizations do not have a technology plan and adopt information technologies in a pragmatic way. Although the number of initiatives is small, it is worth mentioning the implementation of a web information system that allows to register and to carry out researches of blood donors (Khan \& Qureshi, 2009). The system offers security mechanisms against possible unauthorized access that may alter the data. It is also worth mentioning the study developed by the Institute of Fundraising in the United Kingdom that provides a practical guide to selecting and using online giving platforms. In this study, five functions are considered essential (Bloch et al., 2014): (i) feedback to donors; (ii) usability; (iii) donor care; (iv) donor development; and (v) query resolutions. Finally, another guide proposed by Heyman (2016) advocates that usability, security and easier to share and market are key elements in an online for nonprofit fundraising application.

\section{Methodology}

\subsection{Functional and Non-functional Requirements}

The requirements are objectives or functionalities that the client wants to be implemented in a software project. Software requirements are divided into functional and non-functional. Functional requirements are the description of the various functions that users need or want to be implemented (Pressman \& Maxim, 2014, Sommerville, 2015).

Figure 1 shows the UML use cases diagram of the application, which specifies each of the functional requirements offered by the system. The user can register on the website. There are two types of registration: one for institutions and another to donors/ individuals. Institutions can create events/causes; donors can view all the causes created by the institutions, in which they can donate products to an institution. Finally, institutions can see the products donated and the place where they are collected. A

International Journal of Web Applications Volume 11 Number 3 September 2019 
code is generated to track them in real time using the application.

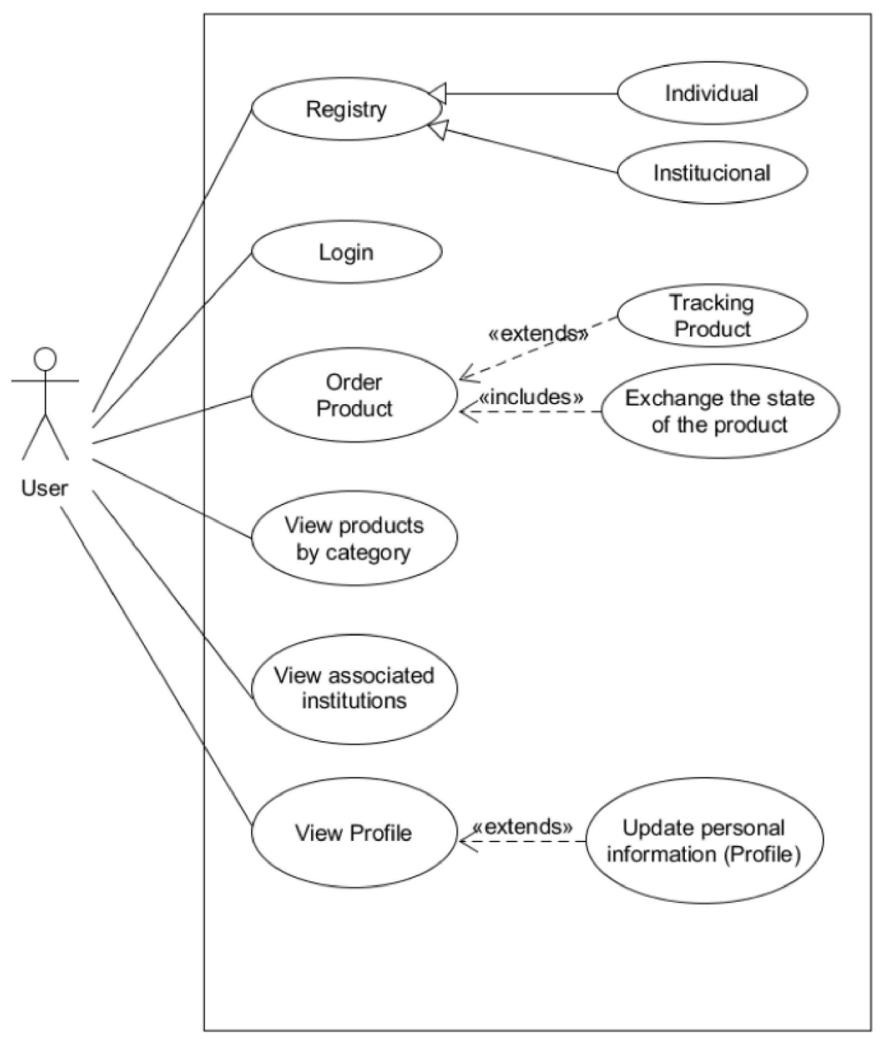

Figure 1. UML use cases

On the other hand, non-functional requirements define the properties and constraints of the system. According to Kassab (2009), non-functional requirements may be even more important than functional requirements because they are common to all system functionalities.

Three non-functional requirements were considered relevant in the context of this project:

-Usability - According to ISO/IEC 9126-1 (2001), usability is defined as "the capability of the software product to be understood, learned, used and attractive to the user, when used under specified conditions". The platform should be intuitive and simple to navigate. Additionally, good usability practices must be adopted in the construction of web applications;

- Usability - A daily backup will be created for the application. It is guaranteed that companies will not lose data, and previous saved data will be used if needed to guarantee the consistency of the system;

- Performance - The system must be able to take all necessary actions within an acceptable time limit. Therefore, the application must minimizing HTTP requests and minifying HTML, CSS and JavaScript.

\subsection{Modeling and Prototype}

A basic and essential operation offered by the system is the registration of users. The user accesses the registration form by navigating through the website, chooses the right form (institutional or an individual user), fills the fields of the form and confirms the operation. This process is modeled in Figure 2.

Institutional users can create causes to be supported by donors. For that, institutional users must fill a form with the information necessary for the cause. At any time, they can update or even delete the cause. Update functionality allows institutional users to change some incorrect information about the cause, and the delete functionality turns the cause inaccessible changing its

\begin{tabular}{lllllll}
\hline 100 & International Journal of Web Applications & Volume & 11 & Number & 3 & September 2019 \\
\hline
\end{tabular}




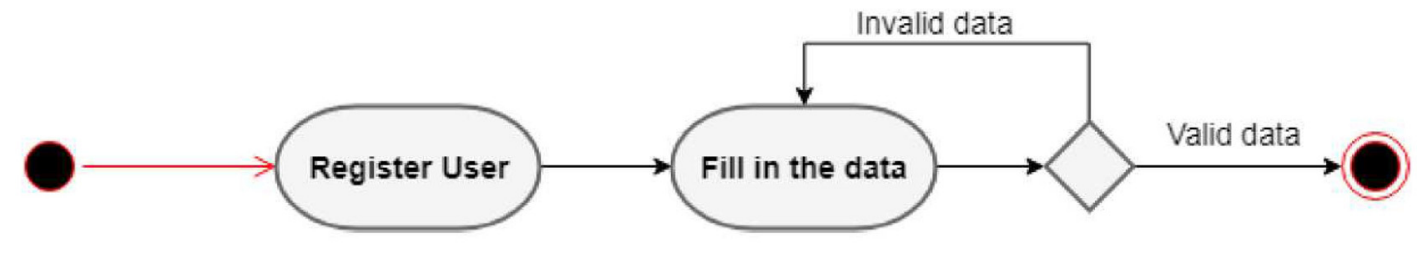

Figure 2. Activity diagram: registration process

state to " 0 ". It should be mentioned that the causes are not deleted from the database, but only their state is changed. This process can be depicted in figure 3 .

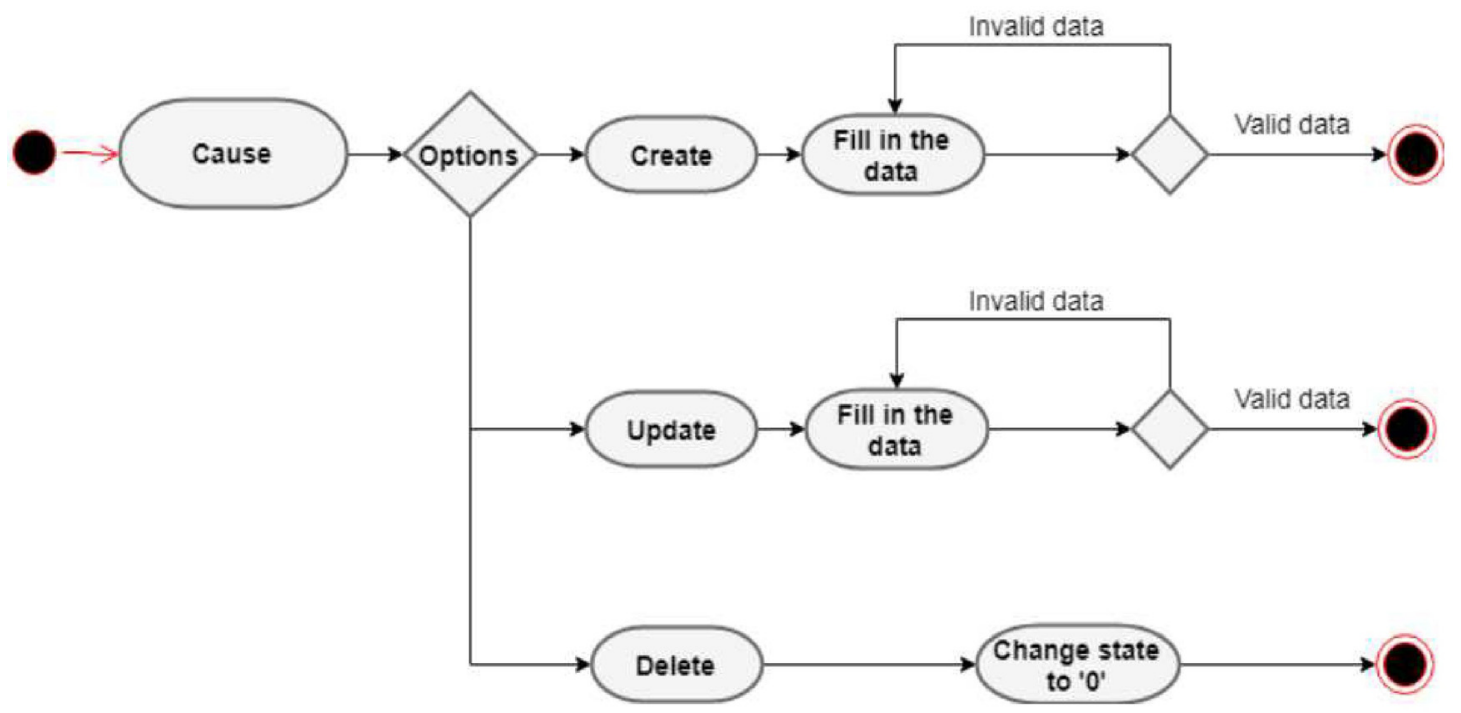

Figure 3. Activity diagram: Creation and management of causes

Individual users or donors can donate and support the cause they want. For that, the user can add new goods filling the data necessary and associating it to a created cause by one institution. Like institutions, individual users can also update or even delete goods/products. The products are also not deleted from the database, but only marked as "deleted" changing their state to " 0 ".

Institutional users can check-out their orders (donated products). After that, a code associated with the order is generated. This information is saved in the database, and the main page is updated. At this point, they don't have products to order, but they can track their pending products. This process is illustrated in Figure 4.

\subsection{Technological Architecture}

The physical architecture presented in Figure 5 is exclusively based on open source technologies and supported on a three layer model: (i) database layer; (ii) business logic layer; and (iii) presentation layer. In the database layer, we adopted the MySQL as a Database Management Systems (DBMS). The business logic layer is responsible for the technical implementation of the system functions, to perform the respective queries to the database and send them to the higher layer. Finally, in the presentation layer, we use HTML5, JavaScript in particular through we used the jQuery library to perform client-side validation of data and Cascade Style Sheets (CSS) are used to define the presentation of pages, which includes the Web layout, fonts type and size, and buttons dimensions.

It is also relevant to model the logical architecture of the system. For this purpose a UML component diagram was used, as shown in Figure 6. The first component that is presented to the user is the "login.php", which is responsible for the authentication process. After that, we have access to four main components: (i) "profile.php" in which the profile of institutions and donors is accessed; (ii) "search_causes.php" which makes possible to search for causes by donors; (iii) "create_cause.php" used by 


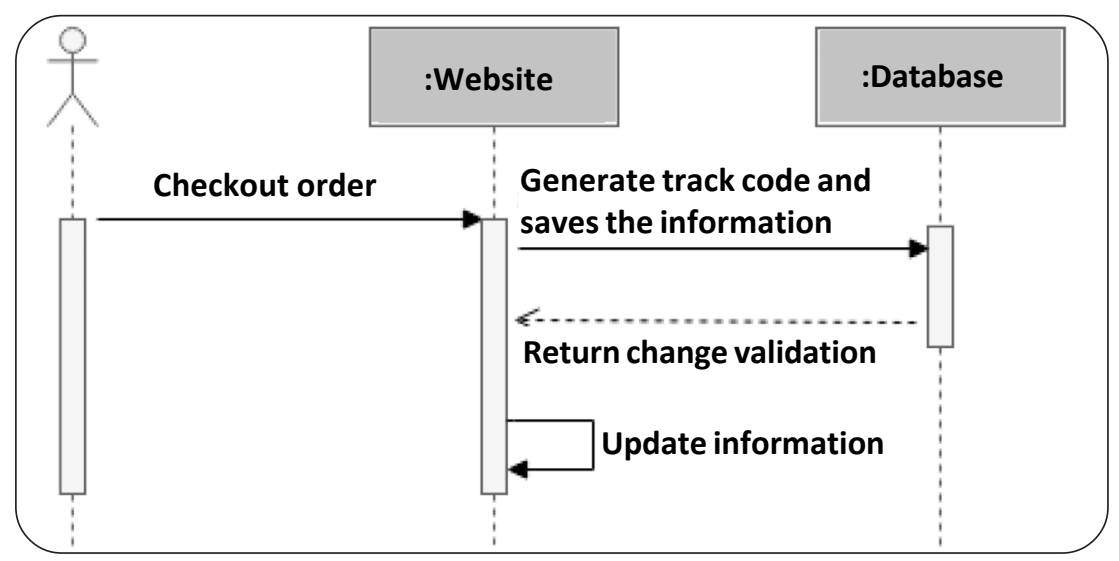

Figure 4. Sequence diagram: Check-out order

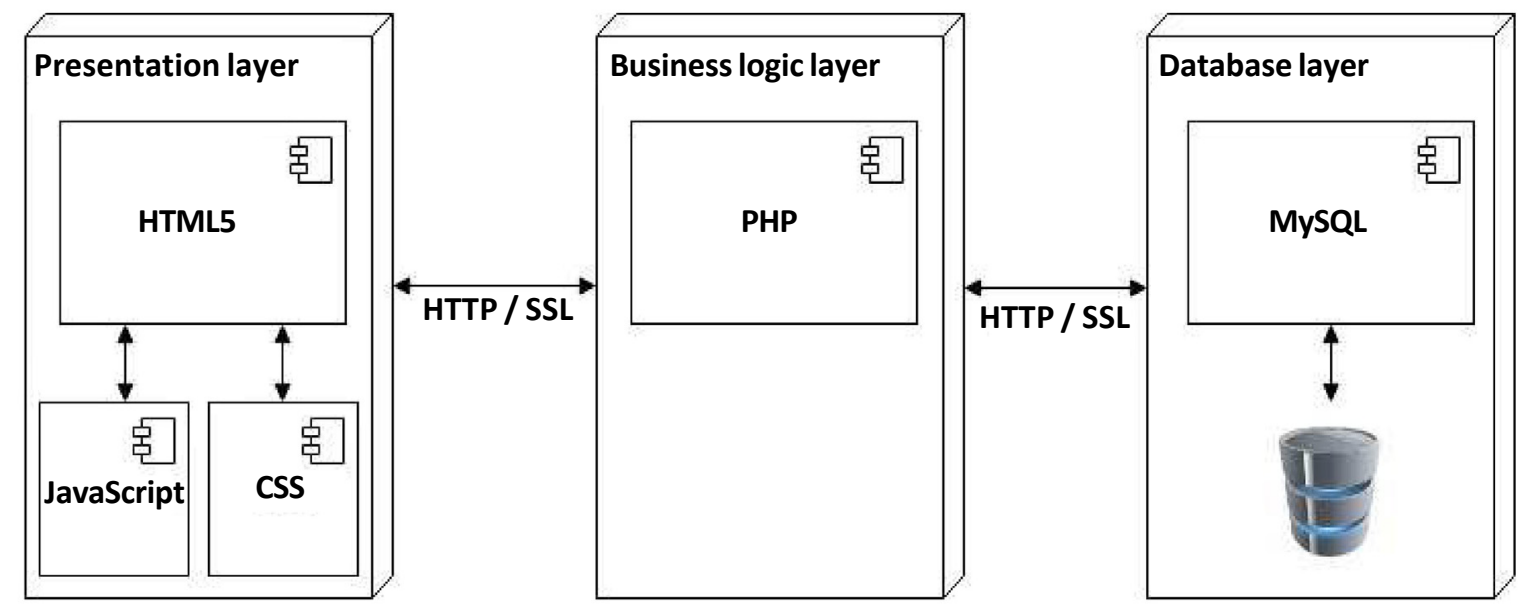

Figure 5. Physical architecture

institutions to create new causes; and (iv) "donate_product.php" through which donors can donate products. It should be mentioned that associated with these main components others PHP classes appear, such as "track_order.php" in which it becomes possible to perform real-time monitoring of orders.

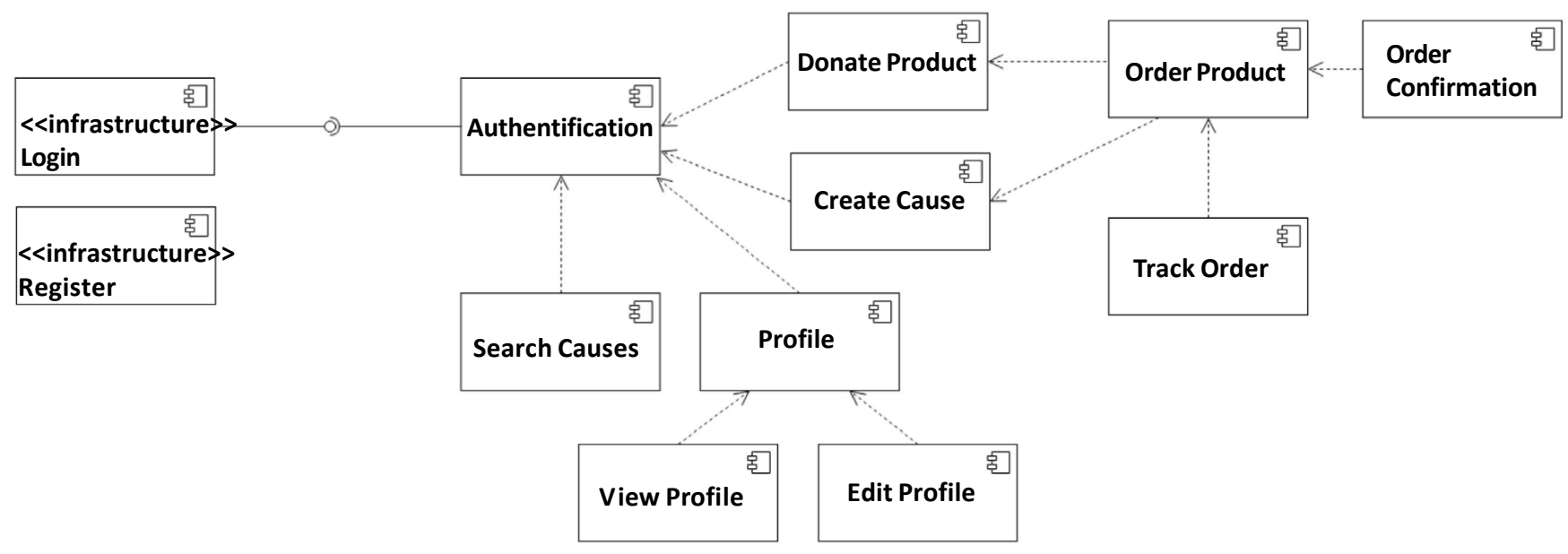

Figure 6. Logical architecture 


\subsection{Database Structure}

Another relevant aspect in the creation of the application was the definition of its data model that can be depicted in Figure 7. There are three fundamental tables: (i) users; (ii) events; and (iii) products. In the table "users" is kept information about each user, being important to distinguish whether it is individual or institutional. In the "events" table, there is information about each cause created by institutional users. The "product" table records the donation of goods made by each individual user. The other three tables turn possible to associate a category to the donated products and allow a user to donate various goods to a cause with different quantities.

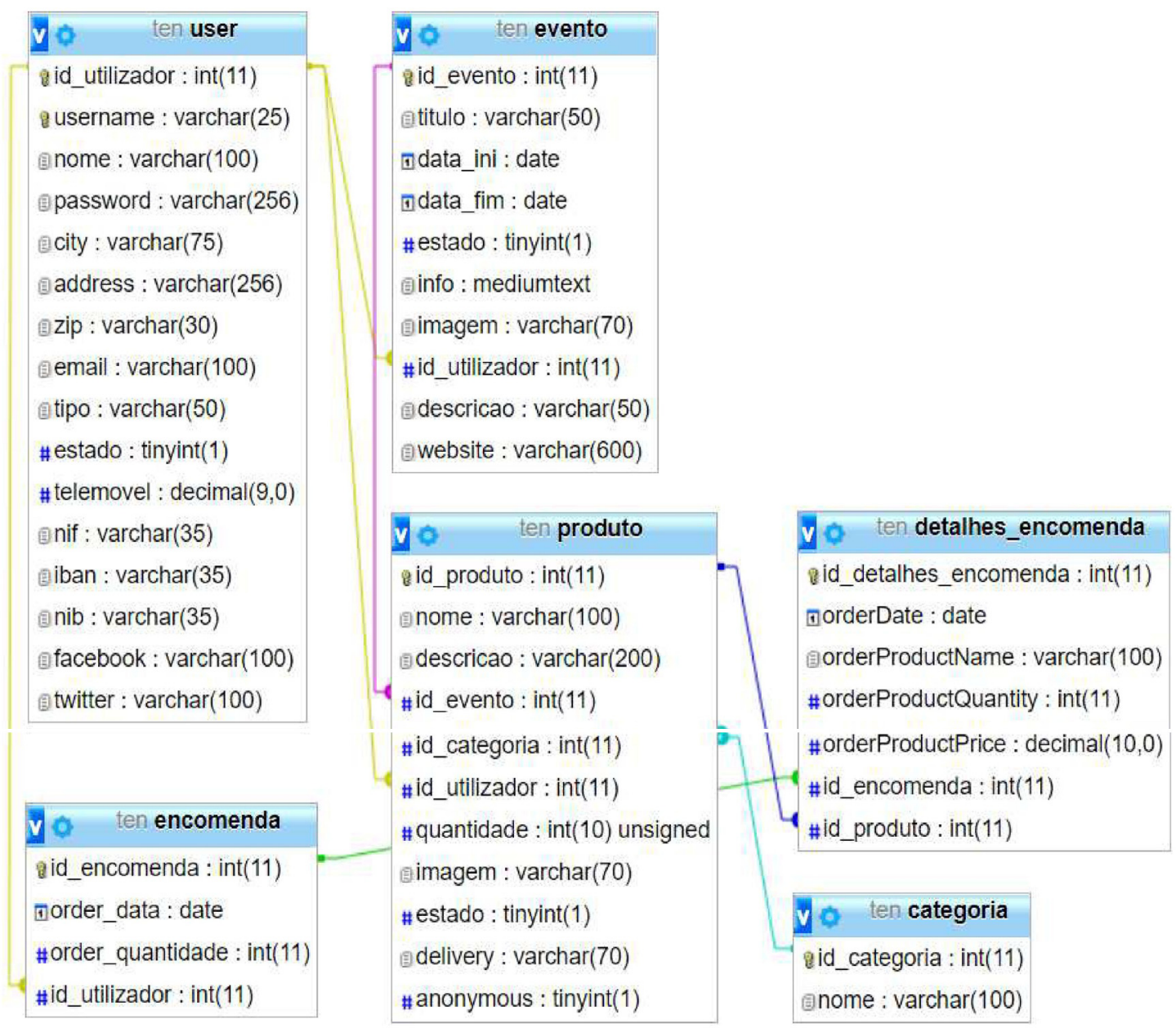

Figure 7. Class model

\subsection{Research Questions}

The research questions were defined based on good practices in the implementation and use of an online grant platform proposed by Bloch et al. (2014) and Heyman (2016). Additionally, the technical practices implemented in the project for nonprofit organizations carried out by Khan \& Qureshi (2009) were also considered. Thus, the following research questions were established:

- RQ1 - Does the application provides feedback to donors?

- RQ2 - Is it possible to tailor the communications and information people receive? Can donors contact the charity directly?

-RQ3 - Do charities received the information/data needed to follow up with donors?

- RQ4 - Is donor anonymity functionality needed?

- RQ5 - How easy is the navigation of the Web application?

International Journal of Web Applications Volume 11 Number 3 September 2019 


\section{Analysis and Discussion of Results}

\subsection{RQ1 - Does the application provide feedback to donors and institutions?}

On the main Web page of the application (homepage) we perform a presentation of the mission, vision and value proposition of the organization. Additionally, we provide a brief contextualization of the project objectives. Also in this Web page, a contact form is available, which allows both the donors and the institution to contact the promoters of the project. This contact can be done both in person and exclusively in an electronic way. In the latter case, the communication is performed using a contact form in which the following information is requested: (i) name; (ii) email; and (iii) message or comment.

4.2 RQ2 - Is it possible to tailor the communications and information people receive? Can donors contact the charity directly? Donors can contact charity organizations directly. For this purpose they must access to the contact information available for each charity organization. In the profile section (Figure 8), each organization provides the following information: (i) website; (ii) facebook page; (iii) twitter; and (iv) physical address. These elements are not mandatory, since we consider that not all organizations can have this information, such as presence on social networks. In any case, and whenever possible, we encourage charity organizations to provide as many contact points as possible.

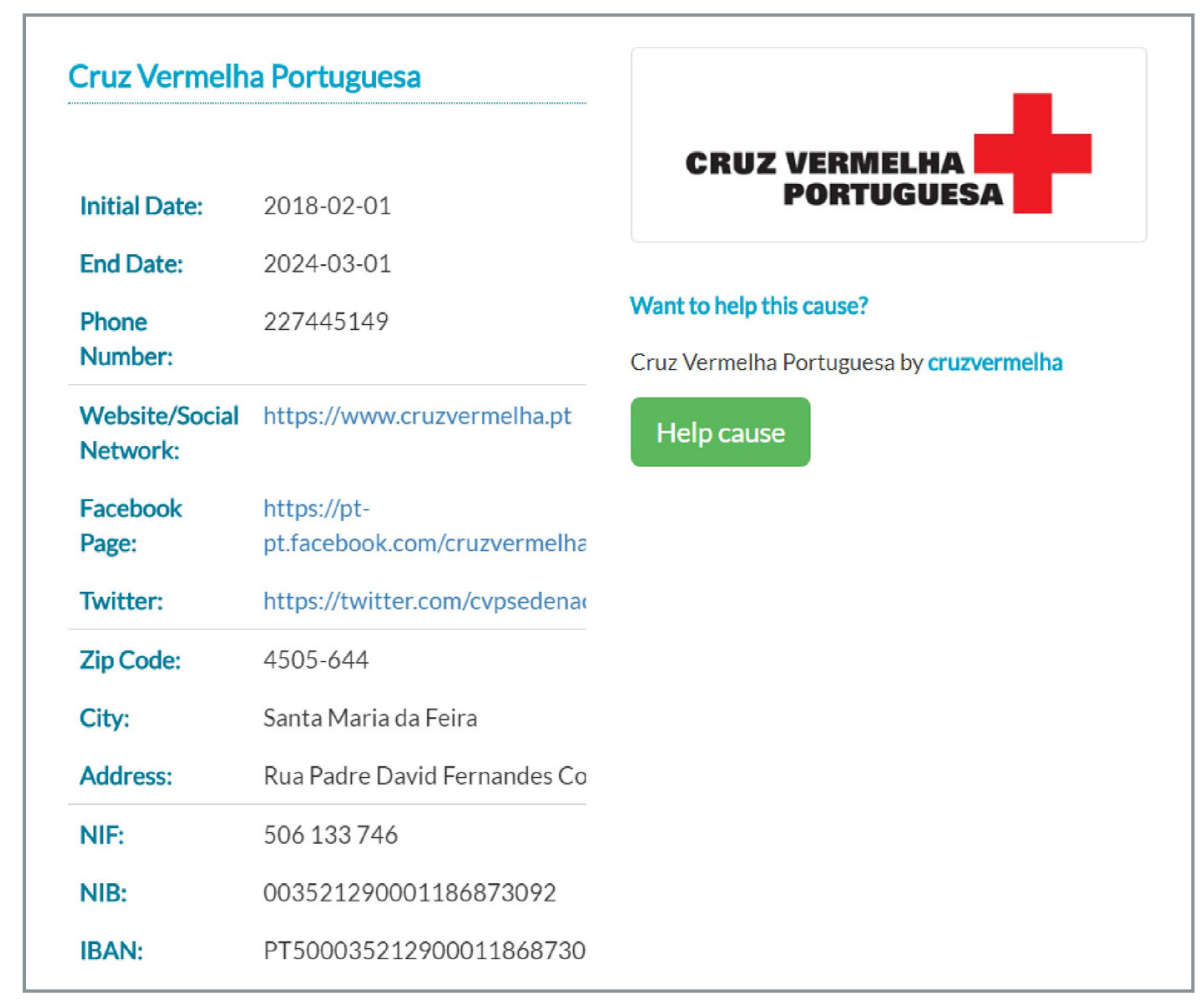

Figure 8. Profile of charity organizations

\subsection{RQ3 - Do charities received the information/data needed to follow up with donors?}

One of the most important features offered by the application is to allow charity organizations to consult the goods donated by donors. The application (Figure 9) distinguishes two stages in the process of donating goods:

1) Pending products - products donated by donors, but which are not yet delivered to the charity organization. The process of collecting the goods can be carried out directly by the charity organization in the company's warehouse or can be requested external services from a transportation company. In this case, the delivery code is presented to the user, so that the charity organization can monitor the delivery of the products in real-time. This process of real-time tracking of deliveries is already carried out by the main players in the transportation sector, such as CTT Expresso, FedEX or UPS;

2) Delivered products - products that are already delivered to the charity organization. Delivery status changes to "delivered".

\begin{tabular}{lllllll}
\hline 104 & International Journal of Web Applications & Volume & 11 & Number & 3 & September 2019 \\
\hline
\end{tabular}


It should also be mentioned that organizations can consult the donor profile in the application. However, if the donation is anonymous then the profile query of the donor is inactive. Instead the information "Anonymous user" is displayed in the Web page.

\begin{tabular}{|c|c|c|c|}
\hline \multicolumn{1}{|c|}{ Pending Products: } \\
\hline Product Name & Donator & Quantity & Delivery Code \\
\hline roupateste & Anonymous User & 2 & PT5a8c65d16acc7 \\
\hline Product Name & Delivered Products: & \\
\hline roupa & Donator & Quantity & Delivery Status \\
\hline cadeirade rodas & alex & 3 & Delivered \\
\hline
\end{tabular}

Figure 9. Donation of goods

\subsection{RQ4 - Is donor anonymity functionality needed?}

Anonymity is referred to by several studies as one of the important elements in the donation process (Perrine \& Heather, 2000; Haley \& Fessler, 2005; Levine \& Johnston, 2014). The role of anonymity is seen as a psychological and social factor among donors and social solidarity entities.

In this sense, the application ensures that donor data are managed according to strict confidentiality criteria under the current legislation. In addition, donations may also be anonymous. To this end, the donor must mark the checkbox "Anonymous Donation" when submitting a new donation. This situation is depicted in Figure 10. We also guarantee that in the database there is no registration of the user associated with the products donated, in the case of an anonymous donation.

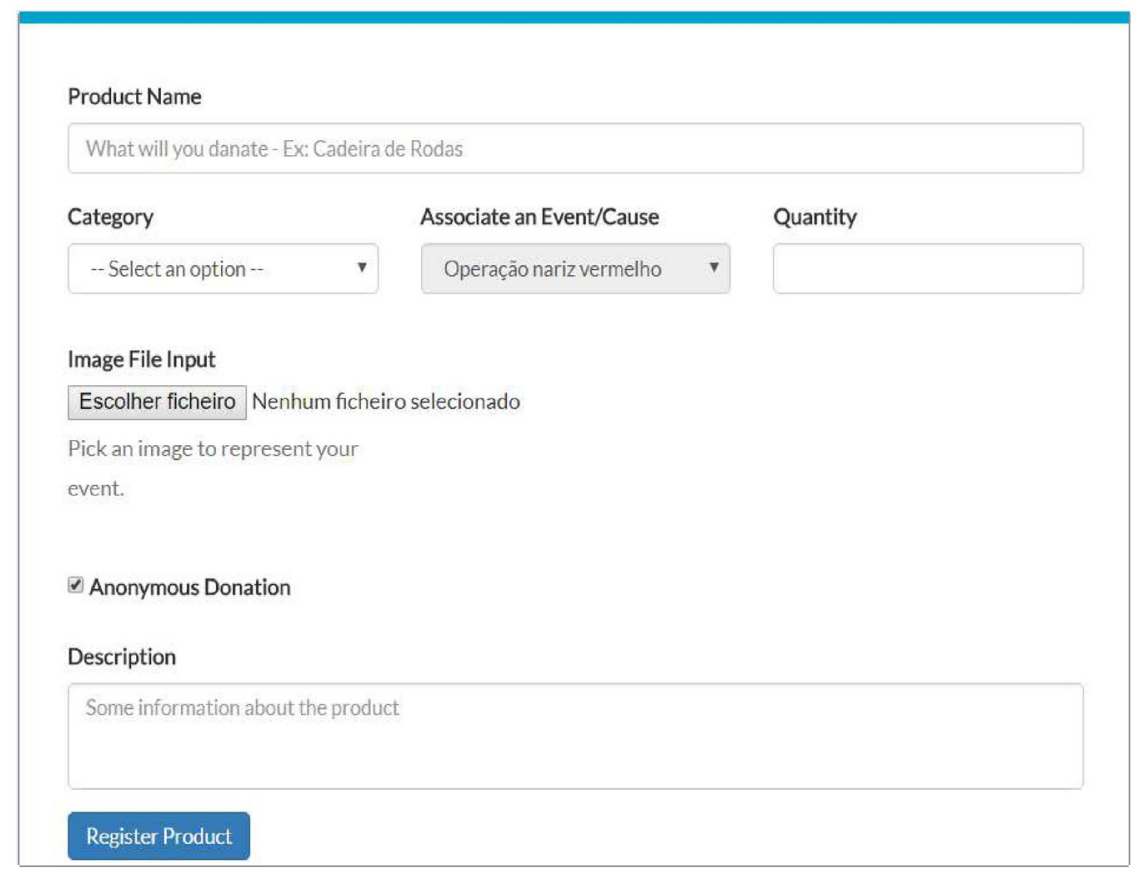

Figure 10. Creating a new donation 


\subsection{RQ5 - How easy is the navigation in the Web application?}

Designing a user interface is critical to the success of a Web application. An interface that is difficult to use can cause users to quit using it and can cause data entry errors and system crashes (Sommerville, 2015). In this way, the design of a Web application should not be limited to the perspective of software engineering, but should also look to its usability a, user interaction and user experience (Almeida \& Monteiro, 2017; Zaror et al., 2018).

In the context of our project, we have adopted the standards defined by the Web Content Accessibility Guideline (WCAG 2.01) according to its four principles:

- Perceivable - information and user interface components must be presentable to users in ways they can perceive;

- Operable - user interface components and navigation must be operable;

- Understandable - information and the operation of user interface must be understandable;

- Robust - content must be robust enough that it can be interpreted reliably by a wide variety of user agents, including assistive technologies.

Additionally, several key design elements proposed by Garett et al. (2017) were adopted:

- Navigation - we chose to use a navigation bar at the top of the page which offers consistency of navigation. All features are accessed through the menu with minimal backtracking/clicks;

- Graphical representation - we used high resolution images of adjustable size, depending on the user's browser and access device. For this purpose, the bootstrap ${ }^{1}$ was adopted as the responsive design framework. Different logos associated with each institution were used, so that donors could more easily identify the social solidarity associations they intend to help. A soft color scheme was also employed with an effective use of white space to avoid visual overload;

- Organization - we always sought to offer an understandable structure of menu functionality. The information was categorized according to the profile of each user (i.e., institutions or donors);

- Content utility - in order for the project to attract donors' interest, it is necessary to have a high degree of commitment from institutions. A greater potential interest from the community can be reached if there are more causes registered in the system. Furthermore, in order to guarantee a high content quality of the causes, each cause submitted by institutions must be previously approved by the administrators of the application. Additionally, we must have up-to-date information. For this, we guarantee that each cause always has a start and end date associated with it. Non-active causes cannot be searchable by donors;

- Purpose - in the current context support for causes of social solidarity is a primary objective of social integration. To this end, the application brings institutions that support these initiatives closer to the people. Another concern that arises in these initiatives and that often inhibits civic participation is the guarantee that the donated assets actually reach the organizations that implement these actions on the ground. These two fundamental goals are achieved with this application;

- Simplicity - we sought to ensure an optimized design of the Web page through the adoption of a responsive design template. Additionally, the time required to find each cause is reduced, since by default the causes are sorted by their end date. Causes that are about to be concluded necessarily deserve greater donor attention;

- Readability - all content is easy to read and intuitive. Each menu of the application offers reduced content avoiding navigation through the scroll bar. Only the "home" page has more than one content block. To facilitate navigation on this page we adopted a single-page application (SPA) approach. According to Joseph (2015), SPA offers high user experience level comparing to classical web applications approach.

\footnotetext{
${ }^{1}$ https://www.w3.org/TR/WCAG20/
}

${ }^{2}$ https://getbootstrap.com

\begin{tabular}{llllll}
\hline 106 & International Journal of Web Applications & Volume 11 & Number 3 & September 2019 \\
\hline
\end{tabular}




\section{Conclusions}

The application helps approaching institutions and donors. Through its use, the donations' process becomes simpler, practical and intuitive for both. For institutions it becomes easier to add new causes and follow the process of donating goods. Donors, for their part, can see causes and donate goods online.

The application provides feedback to both donors and institutions. A contact form has been developed for this purpose. Donors can follow the donation process online, and there is confirmation that the donations have reached their respective institutions. Additionally, donations can also be anonymous, which is an important element in the process of encouraging new donations.

Finally, usability was considered as a fundamental element in the design of the application. We adopted the standards WCAG 2.0 regarding perceivability, operability, understandability, and robustness. Furthermore, other good practices in the construction of Web interfaces were followed, respectively in terms of navigability, organization of elements, utility of contents, simplicity and readability.

As future work, we intend to evaluate the use of this application by the community, in order to identify improvement points and assess the impact of the application to the community of social solidarity institutions and donors. Additionally, we also consider relevant the development of a mobile application for Android and iOS devices.

\section{References}

[1] ABC (2017). Portugal fires: Interior Minister resigns as death toll passes 100, PM under fire to stand down. Retrieved February 28, 2018, from http://www.abc.net.au/news/2017-10-19/portuguese-minister-resigns-as-wildfire-toll-passes-100/9065714

[2] Almeida, F., Monteiro, J. (2017). Approaches and Principles for UX Web Experiences. International Journal of Information Technology and Web Engineering, 12 (2) 49-65.

[3] Bennett, R. (2009). Impulsive donation decision during online browsing of charity websites. Journal of Consumer Behavior, 8(Mar-June), 116-134.

[4] Bloch, A., Drummond, C., Framjee, P. (2014). A practical guide to selecting and using online giving platforms. Institute of Fundraising. Retrieved March 2, 2018, from https://www.institute-of-fundraising.org.uk/library/onlinegiving/makingthemostof digitaldonations.pdf

[5] Boles, B. (2013). Technology's Role in the Nonprofit Sector: Increasing Organizational Effectiveness and Efficiency through Technology Innovations. Columbia Social Work Review, 4, 69-79.

[6] Cacija, L. (2016). The Nonprofit Marketing Process and Fundraising Performance of Humanitarian Organizations: Empirical Analysis. Management, 21(2) 1-25.

[7] Choi, B., Kim, M. (2016). Donation via Mobile Applications: A Study of the Factors Affecting Mobile Donation Application Use. International Journal of Human-Computer Interaction, 32 (12) 967-974.

[8] Cole, C. (2014). Social Media Best Practices for Nonprofit Organizations. Canadian Coalition for Global Health Research. Retrieved March 2, 2018, from http://www.ccghr.ca/wp-content/uploads/2014/06/CCGHR-Social-Media_Introduction-toReport.pdf

[9] Days, F. (2009). How to make social-media fundraising work for you. Nonprofit World, 27 (2) 20.

[10] European Comission (2017). Social economy in the EU. Retrieved February 28, 2018, from http://ec.europa.eu/growth/ sectors/social-economy_pt

[11] Garett, R., Chiu, J., Zhang, L., Young, S. (2017). A Literature Review: Website Design and User Engagement. Online Journal of Communication Media Technology, 6 (3) 1-14.

[12] Hackler, D., Saxton, G. (2007). The Strategic Use of Information Technology by Nonprofit Organizations: Increasing Capacity and Untapped Potential. Public Administration Review, 67 (3) 474-487.

[13] Hagenbuch, D. (2007). Easy Ways to Turn Your Supporters into Fundraisers. Nonprofit World, 25 (1) 22-23.

[14] Haley, K., Fessler, D. (2005). Nobody's watching? Subtle cues affect generosity in an anonymous economic game. Evolution

International Journal of Web Applications Volume 11 Number 3 September 2019 
and Human Behavior, 26, 245-256.

[15] Heyman, D. (2016). Nonprofit Fundraising 101: A Practical Guide to Easy to Implement ideas and Tips from Industry Experts. New Jersey: Wiley.

[16] ISO/IEC 9126. (2001). Quality Characteristics and Guidelines for the User. International Organization for Standardization, Geneva. Retrieved March 3, 2018, from https://webstore.iec.ch/preview/info_isoiec9126-1\%7Bed1.0\%7Den.pdf

[17] Joseph, R. (2015). Single Page Application and Canvas Drawing. International Journal of Web \& Semantic Technology (IJWesT), 6(1) 29-37.

[18] Kassab, M. (2009). Non-Functional Requirements: Modeling and Assessment. Saarbrücken: VDM Verlag.

[19] Khan, A., Qureshi, M. (2009). Web-Based Information System for Blood Donation. International Journal of Digital Content Technology and its Applications, 3 (2) 137-142.

[20] Leskovec, D. (2010). How to Collect Donations: Conceptual Review and Implications for Online Non-Profit Information Goods Providers. Akademija, 55-68.

[21] Lovejoy, K., Saxton, G. (2012). Information, Community, and Action: How Nonprofit Organizations Use Social Media. Journal of Computer-Mediated Communication, 17, 337-353.

[22] Levine, B., Johnston, M. (2014). Campaign contributions should be anonymous. Retrived March 25, 2018, from https:// www.washingtonpost.com/opinions/making-campaign-contributions-anonymous/2014/09/04/65f2b8d8-2e39-11e4-9b98848790384093 story.html?utm_term $=.67 \mathrm{c} 5 \mathrm{c} 19 \mathrm{f} 42 \mathrm{e} 3$

[23] Lucas, E. (2017). Reinventing the rattling tin: How UK charities use Facebook in fundraising. International Journal of Nonprofit and Voluntary Sector Marketing, 22 (2) 1-9.

[24] Mirza, A. (2010). Failure and Success Factors of an Information System Development in a Charitable Organization. Global Journal of Management and Business Research, 10 (3) 79-83.

[25] Moraru, S. (2014). Emerging Trend: the Use of Mobile Technologies in Romanian Nonprofits. In: Proceedings of Conference: Strategica 2014, Bucharest, Romania, 1-16.

[26] Nah, S., Saxton, G. (2013). Modeling the adoption and use of social media by nonprofit organizations. New Media \& Society, 15 (2) 294-313.

[27] Perrine, R., Heather, S. (2000). Effects of Picture and Even-A-Penny-Will-Help Appeals on Anonymous Donations to Charity. Psychological Reports, 86, 551-559.

[28] Pressman, R., Maxim, B. (2014). Software Engineering: A Practitioner's Approach. New York: McGraw-Hill Education.

[29] Ramos, A. (2016). Qual o impacto da economia social em Portugal? Retrieved February 28, 2018, from https://www.tsf.pt/ economia/interior/qual-o-impacto-da-economia-social-em-portugal-5179159.html

[30] Sargeant, A., West, D. C., Jay, E. (2007). The Relational Determinants of Nonprofit Web Site Fundraising Effectiveness: An Exploratory Study. Nonprofit Management \& Leadership, 18 (2) 141-156.

[31] Schwedel, M. (2013). Nonprofit Organizations, Social Media, and Mobile Applications. Master of Public Administration, Department of Political Science in the Graduate School Southern Illinois University Carbondale. Retrieved March 2, 2018, from http://opensiuc.lib.siu.edu/cgi/viewcontent.cgi?article=1583\&context=gs_rp

[32] Sommerville, I. (2015). Software Engineering. London: Pearson Education.

[33] Tatham, P., Christopher, M. (2014). Humanitarian Logistics: Meeting the Challenge of Preparing for and Responding to Disasters. London: Kogan Page.

[34] Tiago, L. (2017). Empreendedorismo, boas práticas empresariais e financiamento são os temas em destaque na $2^{\mathrm{a}}$ edição do Fórum Portugal Economia Social. Retrieved February 28, 2018, from https://www.dinheirovivo.pt/economia/260-mil-trabalhadoresdedicados-fazem-da-economia-social-o-3o-empregador/

[35] Treiblmaier, H., Pollach, I. (2008). Drivers and Inhibitors of Online Donations to Nonprofit Organizations. Journal of International Technology and Information Management, 17 (2) 85-98.

[36] Tugrul, T., Lee, E. (2018). Promoting charitable donation campaigns on social media. The Service Industries Journal, 38 (34) $149-163$.

\begin{tabular}{lllllll}
\hline 108 & International Journal of Web Applications & Volume & 11 & Number & 3 & September 2019 \\
\hline
\end{tabular}


[37] Umapathy, K., Huang, H. (2015). A preliminary Study of Information Technologies Usage in Nonprofit Organizations. In: Proceedings of the Twentieth Americas Conference on Information Systems, Puerto Rico, 1-13.

[38] Warner, T., Abel, A., Hachtmann, F. (2014). Empowered and engaged: Exploring social media best practices for nonprofits. Journal of Digital \& Social Media Marketing, 1 (4) 83-96.

[39] Zarour, M., Alharbi, M., Park, E. (2018). User experience framework that combines aspects, dimensions, and measurement methods. Cogent Engineering, 4 (1) 1-25. 